\title{
Nucleation of $\mathrm{Al}_{3} \mathrm{Zr}$ and $\mathrm{Al}_{3} \mathrm{Sc}$ in aluminum alloys: from kinetic Monte Carlo simulations to classical theory.
}

\author{
Emmanuel Clouet, ${ }^{1,2, \text { * Maylise Nastar, }}{ }^{2}$ and Christophe Sigli $^{1}$ \\ ${ }^{1}$ Pechiney Centre de Recherches de Voreppe, B.P. 27, 38341 Voreppe cedex, France \\ ${ }^{2}$ Service de Recherches de Métallurgie Physique, CEA/Saclay, 91191 Gif-sur-Yvette, France
}

(Dated: November 9, 2018)

\begin{abstract}
$\mathrm{Zr}$ and Sc precipitate in aluminum alloys to form the compounds $\mathrm{Al}_{3} \mathrm{Zr}$ and $\mathrm{Al}_{3} \mathrm{Sc}$ which for low supersaturations of the solid solution have the $\mathrm{L}_{2}$ structure. The aim of the present study is to model at an atomic scale this kinetics of precipitation and to build a mesoscopic model based on classical nucleation theory so as to extend the field of supersaturations and annealing times that can be simulated. We use some ab-initio calculations and experimental data to fit an Ising model describing thermodynamics of the Al-Zr and Al-Sc systems. Kinetic behavior is described by means of an atom-vacancy exchange mechanism. This allows us to simulate with a kinetic Monte Carlo algorithm kinetics of precipitation of $\mathrm{Al}_{3} \mathrm{Zr}$ and $\mathrm{Al}_{3} \mathrm{Sc}$. These kinetics are then used to test the classical nucleation theory. In this purpose, we deduce from our atomic model an isotropic interface free energy which is consistent with the one deduced from experimental kinetics and a nucleation free energy. We test different mean-field approximations (Bragg-Williams approximation as well as Cluster Variation Method) for these parameters. The classical nucleation theory is coherent with the kinetic Monte Carlo simulations only when CVM is used: it manages to reproduce the cluster size distribution in the metastable solid solution and its evolution as well as the steady-state nucleation rate. We also find that the capillary approximation used in the classical nucleation theory works surprisingly well when compared to a direct calculation of the free energy of formation for small $\mathrm{L}_{2}$ clusters.
\end{abstract}

\section{INTRODUCTION}

Precipitation kinetics of a metastable solid solution is known to be divided in three successive stages: the nucleation, growth, and coarsening of nuclei of the new stable phase. The first stage of precipitation is of great practical interest but difficult to observe experimentally. Kinetic Monte Carlo simulation is the suitable tool for a numerical prediction of a nucleation kinetics 1.2 but a rationalization of the results is difficult and atomic simulations cannot reach very low supersaturations. On the other hand, classical descriptions of these different stages 3.4 are well established and the associated models are now widely used to understand experimental kinetics and to model technological processes $5,6,7,8$. Recently, classical nucleation theory has been shown to be in good agreement with more reliable atomic models by way of a direct comparison with kinetic Monte Carlo simulations 9.10.11.12.13. These different studies included decomposition of a metastable solid solution for a demixing binary system on a surface ${ }^{9}$ or in the bulk ${ }^{11,12}$ and kinetics of electrodeposition on a surface ${ }^{13}$. In this last study, Berthier et al. show that physical parameters of classical nucleation theory have to be carefully calculated so as to reproduce atomic simulations. In the present paper, we want to extend the range of comparison between classical nucleation theory and atomic simulations by studying the case of an ordering system on a frustrated lattice. We thus choose to model kinetics of precipitation of a $\mathrm{L}_{2}$ ordered compound formed from a solid solution lying on a face centered cubic (fcc) lattice.

For fcc lattice it is now well established that one has to use a mean field approximation more accurate than the widely used Bragg-Williams one in order to calculate thermodynamic properties ${ }^{14}$. The Cluster Variation Method $(\mathrm{CVM})^{15.16}$ enables one to obtain phase diagrams which are in quantitative agreement with thermodynamic Monte Carlo simulations ${ }^{17.18}$. When CVM is used, frustration effects on the tetrahedron of first nearest neighbors and short range order due to interactions are considered in a satisfying way enabling one to predict quantitatively thermodynamic behavior. Nevertheless, the use of CVM is often restricted to the calculation of equilibrium properties and, thus, for computing thermodynamic properties of the metastable supersaturated solid solution in classical nucleation theory one merely considers Bragg-Williams approximation. The purpose of this paper is then to show that the use of CVM calculations with classical nucleation theory leads to a satisfying description of the metastable solid solution and extend the range of supersaturations that can be modelled with this theory.

In this purpose we build an atomic model which allows us to study kinetics of precipitation of $\mathrm{Al}_{3} \mathrm{Zr}$ and $\mathrm{Al}_{3}$ Sc. The two considered binary systems, Al-Zr and Al-Sc, have different kinetic properties: the interaction with vacancies is repulsive for $\mathrm{Zr}$ atoms whereas it is attractive for Sc atoms. On the other hand, for low supersaturations, thermodynamics of both systems are quite similar. $\mathrm{Al}_{3} \mathrm{Zr}$ has the stable $\mathrm{DO}_{23}$ structure ${ }^{19}$, but for small supersaturations of the solid solution, $\mathrm{Al}_{3} \mathrm{Zr}$ precipitates with the metastable $\mathrm{L}_{2}$ structure and precipitates with the $\mathrm{DO}_{23}$ structure only appear for prolonged heat treatment and high enough supersaturations 6.20.21. As for $\mathrm{Al}_{3} \mathrm{Sc}$, the stable structure is $\mathrm{L}_{2}{ }^{19}$ and thus only $\mathrm{L}_{2}$ precipitates have been observed during experimen- 
tal kinetics ${ }^{22,23,24}$. In this study we mainly focus on the nucleation stage and therefore we consider that both $\mathrm{Zr}$ and Sc lead to the precipitation of a compound having the $\mathrm{L}_{2}$ structure. In this context, Al-Zr and Al-Sc systems are really similar from a thermodynamic point of view unlike their kinetic behavior. It is then interesting to study these two systems in parallel and to see if classical nucleation theory manages to reproduce atomic simulations for these two different kinetic behaviors.

The atomic model used in kinetic Monte Carlo simulations is built using experimental data as well as ab-initio calculations. We deduce from it physical parameters entering mesoscopic models like classical nucleation theory and show how this theory compares to atomic simulations for different supersaturations and different annealing temperatures. The capillary approximation used in classical nucleation theory is then discussed as well as different mean field approximations that can be combined with it.

\section{ATOMIC MODEL}

\section{A. Al-Zr and Al-Sc thermodynamics}

In order to simulate thermodynamic behavior of $\mathrm{Al}-\mathrm{Zr}$ and Al-Sc binary systems, we use a rigid lattice: configurations of the system are described by the occupation numbers $p_{n}^{i}$ with $p_{n}^{i}=1$ if the site $n$ is occupied by an atom of type $i$ and $p_{n}^{i}=0$ if not. Energies of such configurations are given by an Ising model with first and second nearest neighbor interactions. This is the simplest model to simulate precipitation of a stoichiometric $\mathrm{Al}_{3} \mathrm{X}$ compound in the $\mathrm{L}_{2}$ structure. Indeed one has to include second nearest neighbor interactions, otherwise $\mathrm{L}_{2}$ precipitates do not show perfect $\mathrm{Al}_{3} \mathrm{X}$ composition. On the other hand, there is no use to consider interactions beyond second nearest neighbors as these interactions are significantly lower than first and second nearest neighbor interactions ${ }^{25}$. We could have considered interactions for clusters other than pairs too, but we showed that the use of interactions for first nearest neighbor triangle and tetrahedron does not really change the kinetics of precipitation 26 : the Onsager coefficients defining diffusion in the solid solution are unchanged with or without these interactions as well as the nucleation free energy. Thus, in our model, the energy per site of a given configuration is

$$
E=\frac{1}{2 N_{s}} \sum_{\substack{n, m \\ i, j}} \epsilon_{i j}^{(1)} p_{n}^{i} p_{m}^{j}+\frac{1}{2 N_{s}} \sum_{\substack{r, s \\ i, j}} \epsilon_{i j}^{(2)} p_{r}^{i} p_{s}^{j}
$$

where the first and second sums respectively runs on all first and second nearest neighbor pairs of sites, $N_{s}$ is the number of lattice sites, $\epsilon_{i j}^{(1)}$ and $\epsilon_{i j}^{(2)}$ are the respective effective energies of a first and second nearest neighbor pair in the configuration $\{i, j\}$.
TABLE I: First and second nearest neighbor pair effective energies (in eV). Only interactions different from zero are presented.

\begin{tabular}{lllll}
\hline \hline$\epsilon_{\mathrm{AlAl}}^{(1)}$ & $=-0.560$ & $\epsilon_{\mathrm{AlV}}^{(1)}$ & -0.222 \\
$\epsilon_{\mathrm{ZrZr}}^{(1)}$ & $=-1.045$ & $\epsilon_{\mathrm{ZrV}}^{(1)}=$ & -0.350 \\
$\epsilon_{\mathrm{ScSc}}^{(1)}$ & $=-0.650$ & $\epsilon_{\mathrm{ScV}}^{(1)}$ & $=-0.757$ \\
$\epsilon_{\mathrm{AlZr}}^{(1)}$ & $=-0.979+24.4 \times 10^{-6} T$ & & \\
$\epsilon_{\mathrm{AlSc}}^{(1)}=-0.759+21.0 \times 10^{-6} T$ & & \\
$\epsilon_{\mathrm{VV}}^{(1)}=-0.084$ & & \\
$\epsilon_{\mathrm{AlZr}}^{(2)}$ & $=+0.101-22.3 \times 10^{-6} T$ & & \\
$\epsilon_{\mathrm{AlSc}}^{(2)}$ & $=+0.113-33.4 \times 10^{-6} T$ & & \\
\hline \hline
\end{tabular}

With such a model, as long as vacancy concentration can be neglected, thermodynamic behavior of Al-X system $(\mathrm{X} \equiv \mathrm{Zr}$ or $\mathrm{Sc}$ ) only depends on the order energies

$$
\begin{aligned}
& \omega^{(1)}=\epsilon_{\mathrm{AlX}}^{(1)}-\frac{1}{2} \epsilon_{\mathrm{AlAl}}^{(1)}-\frac{1}{2} \epsilon_{\mathrm{XX}}^{(1)}, \\
& \omega^{(2)}=\epsilon_{\mathrm{AlX}}^{(2)}-\frac{1}{2} \epsilon_{\mathrm{AlAl}}^{(2)}-\frac{1}{2} \epsilon_{\mathrm{XX}}^{(2)} .
\end{aligned}
$$

We use experimental data combined with ab-initio calculations to obtain these order energies for Al-Zr and Al-Sc systems.

First nearest neighbor order energies are chosen so as to correctly reproduce formation energies of $\mathrm{Al}_{3} \mathrm{Zr}$ and $\mathrm{Al}_{3} \mathrm{Sc}$ compounds in $\mathrm{L}_{2}$ structure, $\Delta F\left(\mathrm{Al}_{3} \mathrm{X}, \mathrm{L}_{2}\right)=$ $3 \omega^{(1)}$. For $\mathrm{Al}_{3} \mathrm{Zr}$, we use the free energy of formation that we previously calculated 25 . For $\mathrm{Al}_{3} \mathrm{Sc}$, we calculate the enthalpy of formation with the full-potential linearmuffin-tin-orbital method ${ }^{27}$ in the generalized gradient $\operatorname{approximation}^{28}$ and we use the value of Ref. 29 and 30 for the vibrational contribution to the free energy of formation:

$$
\begin{aligned}
& \Delta F\left(\mathrm{Al}_{3} \mathrm{Zr}, \mathrm{Ll}_{2}\right)=-0.530+73.2 \times 10^{-6} \mathrm{~T} \mathrm{eV} \\
& \Delta F\left(\mathrm{Al}_{3} \mathrm{Sc}, \mathrm{Ll}_{2}\right)=-0.463+62.9 \times 10^{-6} \mathrm{~T} \mathrm{eV}
\end{aligned}
$$

Second nearest neighbor interactions are chosen so as to reproduce $\mathrm{Zr}$ and $\mathrm{Sc}$ solubility limits in $\mathrm{Al}$. Indeed these limits only depend on order energy $\omega^{(2)}$, as can be seen from the low temperature expansion ${ }^{31}$ to the second order in excitation energies:

$$
x_{\mathrm{X}}^{e q}=\exp \left(-6 \omega^{(2)} / k T\right)+6 \exp \left(-10 \omega^{(2)} / k T\right) .
$$

We check using the CVM in the tetrahedron-octahedron approximation ${ }^{15,16}$ that this low temperature expansion for the solubility limit is correct in the whole range of temperature of interest, i.e. until $\mathrm{Al}$ melting temperature $\left(T^{m e l}=934 \mathrm{~K}\right)$. For Al-Zr interactions, as we want to model precipitation of the metastable $\mathrm{L} 1_{2}$ structure of $\mathrm{Al}_{3} \mathrm{Zr}$ compound, we use the metastable solubility limit that we previously obtained from ab-initio calculations ${ }^{25}$, whereas for $\mathrm{Al}_{3} \mathrm{Sc}$ the $\mathrm{L}_{2}$ structure is stable and we use 
the solubility limit arising from a thermodynamic modelling of experimental data 32 :

$$
\begin{aligned}
x_{\mathrm{Zr}}^{e q} & =\exp \left(\left(-0.620+155 \times 10^{-6} T\right) \mathrm{eV} / k T\right), \\
x_{\mathrm{Sc}}^{e q} & =\exp \left(\left(-0.701+230 \times 10^{-6} T\right) \mathrm{eV} / k T\right) .
\end{aligned}
$$

One should notice that these solubility limits have been found to be consistent with ab-initio calculations 25.30 and thus with the formation energies we used for $\mathrm{Al}_{3} \mathrm{Zr}$ and and $\mathrm{Al}_{3} \mathrm{Sc}$.

Unlike thermodynamics, kinetics does not only depend on order energies but also on effective energies $\epsilon_{i j}^{(1)}$ and $\epsilon_{i j}^{(2)}$. We deduce them from $\omega^{(1)}$ and $\omega^{(2)}$ by using experimental values for cohesive energies of pure elements 33 : $E^{c o h}(\mathrm{Al})=3.36 \mathrm{eV}, E^{c o h}(\mathrm{Zr})=6.27 \mathrm{eV}$, and $E^{c o h}(\mathrm{Sc})=3.90 \mathrm{eV}$. We assume that second nearest neighbor interactions do not contribute to these cohesive energies, i.e. $\epsilon_{\mathrm{XX}}^{(2)}=0(\mathrm{X} \equiv \mathrm{Al}, \mathrm{Zr}$, or $\mathrm{Sc})$ and we neglect any possible temperature dependence of these energies. Therefore, the cohesive energy of $\mathrm{X}$ element is $E^{c o h}(\mathrm{X})=6 \epsilon_{\mathrm{XX}}^{(1)}$. Resulting effective energies are presented in table 【

\section{B. Al-Zr and Al-Sc kinetics}

We introduce in the Ising model atom-vacancy interactions for first nearest neighbors (Tab. II), so as to consider the electronic relaxations around the vacancy. Without these interactions, the vacancy formation energy $E_{\mathrm{V}}^{\text {for }}$ in a pure metal would necessarily equal the cohesive energy which is in contradiction with experimental data. $\epsilon_{\mathrm{AlV}}^{(1)}$ and $\epsilon_{\mathrm{ZrV}}^{(1)}$ are deduced from vacancy formation energy respectively in pure $\mathrm{A}^{134}, E_{\mathrm{V}}^{\text {for }}=0.69 \mathrm{eV}$, and in pure $\mathrm{Zr}^{35}$, $E_{\mathrm{V}}^{f o r}=2.07 \mathrm{eV}$. For $\mathrm{Zr}$, this energy corresponds to the hcp structure which is quite similar to the fcc one (same first nearest neighborhood). Therefore, we assume that the vacancy energy is the same in both structures. This is then possible to correct this formation energy to take into account the difference between $\mathrm{Al}$ and $\mathrm{Zr}$ equilibrium volumes, but this leads to a correction of $\sim 10 \%$ for $E_{\mathrm{V}}^{\text {for }}$ and does not really change the physical interaction between $\mathrm{Zr}$ atoms and vacancies. We thus choose to neglect such a correction. To compute the interaction between Sc atoms and vacancies, we can directly deduce $\epsilon_{\mathrm{ScV}}^{(1)}$ from the experimental binding energy in aluminum ${ }^{36}$, $E_{\mathrm{ScV}}^{b i n}=\epsilon_{\mathrm{AlV}}^{(1)}+\epsilon_{\mathrm{AlSc}}^{(1)}-\epsilon_{\mathrm{ScV}}^{(1)}-\epsilon_{\mathrm{AlAl}}^{(1)}=0.35 \mathrm{eV}$ at $650 \mathrm{~K}$. Such an experimental data does not exist for Zr impurity, but we can check that the physical interaction we obtain is correct. The binding energy deduced from our set of parameters is strongly negative $\left(E_{\mathrm{ZrV}}^{b i n}=-0.276 \mathrm{eV}\right.$ at $650 \mathrm{~K})$. This is in agreement with the experimental fact that no attraction has been observed between vacancy and $\mathrm{Zr}$ impurity ${ }^{34.37}$. This repulsion in the case of $\mathrm{Zr}$ impurity and this attraction in the case of Sc impurity are related to the difference of cohesive energies between $\mathrm{Zr}$
TABLE II: Kinetic parameters: contribution of the jumping atom to the saddle point energy $e_{\alpha}^{s p}$ and attempt frequency $\nu_{\alpha}$ for $\alpha \equiv \mathrm{Al}, \mathrm{Zr}$, and Sc atoms.

\begin{tabular}{rlr}
\hline \hline$e_{\mathrm{Al}}^{s p}=-8.219 \mathrm{eV}$ & $\nu_{\mathrm{Al}}=$ & $1.36 \times 10^{14} \mathrm{~Hz}$ \\
$e_{\mathrm{Zr}}^{s p}=-11.464 \mathrm{eV}$ & $\nu_{\mathrm{Zr}}=$ & $9 \times 10^{16} \mathrm{~Hz}$ \\
$e_{\mathrm{Sc}}^{s p}=-9.434 \mathrm{eV}$ & $\nu_{\mathrm{Sc}}=$ & $4 \times 10^{15} \mathrm{~Hz}$ \\
\hline \hline
\end{tabular}

and Sc, showing thus that elastic relaxations around the vacancy are not the dominant effect. It could explain why $\mathrm{Zr}$ diffusion coefficient in aluminum is so low compared to the Sc one. Some ab-initio calculations have been made to compute this binding energy with a vacancy for all transition metals in aluminum ${ }^{38}$. They obtained in the case of $\mathrm{Zr}$ as well as Sc impurity a repulsive interaction with a vacancy. This is in contradiction with the experimental data we use to compute Sc-vacancy interaction. Such a disagreement may arise from approximations made in the calculation (Kohn-Korringa-Rostoker Green's function method) as the neglect of atom relaxations and the box that includes only the first nearest neighbors of the impurity-vacancy complex. Nevertheless these ab-initio calculations showed that a binding energy as large as $0.35 \mathrm{eV}$ is possible as the value obtained for Sr impurity was even larger.

We use the experimental value of the divacancy binding energy $^{34}, E_{2 \mathrm{~V}}^{b i n}=0.2 \mathrm{eV}$, in order to compute a vacancyvacancy interaction, $\epsilon_{\mathrm{VV}}^{(1)}=2 \epsilon_{\mathrm{AlV}}^{(1)}-\epsilon_{\mathrm{AlAl}}^{(1)}-E_{2 \mathrm{~V}}^{b i n}$. If we do not include this interaction and set it equal to zero instead, we obtain a binding energy which is slightly too low, divacancies being thus not as stable as they should be. Some recent ab-initio calculations 39.40 have shown that divacancies should be actually unstable, the nonArrhenius temperature dependence of the vacancy concentration arising from anharmonic atomic vibrations. Nevertheless, this does not affect our Monte Carlo simulations as we only include one vacancy in the simulation box, but this divacancy binding energy should be considered more seriously if one wants to build a mean field approximation of our diffusion model or if one wants to compensate vacancy trapping by adding new vacancies in the simulation box.

Diffusion is described through vacancy jumps. The vacancy exchange frequency with one of its twelve first nearest neighbors of type $\alpha$ is given by

$$
\Gamma_{\alpha-\mathrm{V}}=\nu_{\alpha} \exp \left(-\frac{E_{\alpha}^{a c t}}{k T}\right),
$$

where $\nu_{\alpha}$ is an attempt frequency and the activation energy $E_{\alpha}^{a c t}$ is the energy change required to move the $\alpha$ atom from its initial stable position to the saddle point position. It is computed as the difference between the contribution $e_{\alpha}^{s p}$ of the jumping atom to the saddle point energy and the contributions of the vacancy and of the jumping atom to the initial energy of the stable position. This last contribution is obtained by considering all bonds which are broken by the jump. 
The attempt frequency $\nu_{\alpha}$ and the contribution $e_{\alpha}^{s p}$ of the jumping atom to the saddle point energy can depend on the configuration 41.42 .43 . Nevertheless, we do not have enough information to see if such a dependence holds in the case of Al-Zr or Al-Sc alloys. We thus assume that these parameters depend only on the nature of the jumping atom. We fit the six resulting kinetic parameters (Tab. III) so as to reproduce $\mathrm{Al}$ self-diffusion coefficient 44 and $\mathrm{Zr}^{44.45}$ and $\mathrm{Sc}^{46}$ impurity diffusion coefficients:

$$
\begin{aligned}
& D_{\mathrm{Al}^{*}}=0.173 \times 10^{-4} \exp (-1.30 \mathrm{eV} / k T) \mathrm{m}^{2} \cdot \mathrm{s}^{-1} \\
& D_{\mathrm{Zr}^{*}}=728 \times 10^{-4} \exp (-2.51 \mathrm{eV} / k T) \mathrm{m}^{2} \cdot \mathrm{s}^{-1}, \\
& D_{\mathrm{Sc}^{*}}=5.31 \times 10^{-4} \exp (-1.79 \mathrm{eV} / k T) \mathrm{m}^{2} \cdot \mathrm{s}^{-1}
\end{aligned}
$$

$\mathrm{Zr}$ is diffusing slower than Sc which itself is diffusing slower than Al. The difference between diffusion coefficients is decreasing with the temperature, but at the maximal temperature we considered, i.e. $T=873 \mathrm{~K}$, it still remains important as we then have $D_{\mathrm{Al}^{*}} \sim 20 D_{\mathrm{Sc}^{*}} \sim$ $2000 D_{\mathrm{Zr}^{*}}$.

\section{Monte Carlo simulations}

We use residence time algorithm to run kinetic Monte Carlo simulations. The simulation boxes contain $N_{s}=$ $100^{3}$ or $200^{3}$ lattice sites and a vacancy occupies one of these sites. At each step, the vacancy can jump with one of its twelve first nearest neighbors, the probability of each jump being given by Eq. (5). The time increment corresponding to this event is

$$
\Delta t=\frac{1}{N_{s}\left(1-13 x_{\mathrm{X}}^{0}\right) C_{\mathrm{V}}(\mathrm{Al})} \frac{1}{\sum_{\alpha=1}^{12} \Gamma_{\alpha-\mathrm{V}}},
$$

where $x_{\mathrm{X}}^{0}$ is the nominal concentration of the simulation box $\left(\mathrm{X} \equiv \mathrm{Zr}\right.$ or $\mathrm{Sc}$ ) and $C_{\mathrm{V}}(\mathrm{Al})$ the real vacancy concentration in pure $\mathrm{Al}$ as deduced from energy parameters of table [ The first factor appearing in Eq. (6) is due to the difference between the experimental vacancy concentration in pure $\mathrm{Al}$ and the one observed during the simulations. The dependence of this factor with the concentration $x_{\mathrm{X}}^{0}$ reflects that for each impurity the corresponding lattice site and its twelve first nearest neighbors cannot be considered as being pure Al. It is correct only for a random solid solution in the dilute limit, but concentrations considered in this study are low enough so the same expression can be kept for this factor. The absolute time scale is then obtained by summing only configurations where the vacancy is surrounded by $\mathrm{Al}$ atoms in its first nearest neighborhood, i.e. where the vacancy is in pure $\mathrm{Al}$. This ensures that the influence on the time scale of the thermodynamic interaction between the vacancy and the Sc or $\mathrm{Zr}$ impurity is correctly taken into account. The method employed here is equivalent to measuring the fraction of time spent by the vacancy in pure $\mathrm{Al}$ during the simulation and multiplying then all time increments by this factor as done in Ref. 43. We check by running Monte Carlo simulations for different sizes of the box that results do not depend on the effective vacancy concentration.

For low impurity concentration, residence time algorithm can be sped up by noticing that in most of the explored configurations the vacancy is located in pure $\mathrm{Al}$, i.e. on a lattice site where all exchange frequencies are equal to the one in pure $\mathrm{Al}$. In such a configuration, the move of the vacancy can be associated to a random walk and the corresponding time increment is known a priori. Lattice sites corresponding to such configurations can be detected in the beginning of the simulation and the corresponding tables need to be modified only each time the vacancy exchanges with an impurity ${ }^{47}$. For impurity concentration in the range $5 \times 10^{-3} \leq x_{\mathrm{X}}^{0} \leq 1 \times 10^{-2}$, the algorithm is sped up by a factor $\sim 2$. This allows us to simulate lower supersaturations of the solid solution than we could have done with a conventional algorithm.

So as to follow kinetics of precipitation in the simulation box, we have to give us a criterion to discriminate atoms belonging to the solid solution from those in $\mathrm{L}_{2}$ precipitates. As, according to the phase diagram, the stoichiometry of theses precipitates is almost perfect, we only look at Zr or Sc atoms and assume for each of these atoms in a $\mathrm{L}_{2}$ cluster that three associated $\mathrm{Al}$ atoms belong to the same cluster. Zr (or Sc) atoms are counted as belonging to a cluster having $\mathrm{L} 1_{2}$ structure if all their twelve first nearest neighbors are $\mathrm{Al}$ atoms and at least one of their six second nearest neighbors is a $\mathrm{Zr}$ (or Sc) atom. This criterion works only for dimers and bigger clusters and then all Zr or Sc atoms not belonging to such clusters are considered to be monomers. We only counted as precipitates $\mathrm{L} 1_{2}$ clusters bigger than a critical size, i.e. containing more $\mathrm{Zr}$ or Sc atoms than a critical number $n_{\mathrm{X}}^{*}$, this critical number being chosen as the initial one given by classical nucleation theory ( $c f$. section III). Clusters smaller than this critical size are unstable and will re-dissolve into the solid solution. Therefore atoms contained in such clusters are counted as belonging to the solid solution. With this criterion, we can measure during the atomic simulations the number of stable precipitates and their mean size (Fig. 1] and 2). The solid solution concentration is then defined at each step by the relation

$$
x_{\mathrm{X}}=\sum_{n_{\mathrm{X}}=1}^{n_{\mathrm{X}}^{*}} n_{\mathrm{X}} C_{n_{\mathrm{X}}}
$$

where $C_{n_{\mathrm{X}}}$ is the number of $\mathrm{L}_{2}$ clusters containing $n_{\mathrm{X}} \mathrm{Zr}$ or Sc atoms normalized by the number of lattice sites, i.e. the instantaneous probability to observe such a cluster in the simulation box.

All starting configurations for simulations are completely disordered (random) solid solutions. We thus simulate infinitely fast quenching from high temperatures. During the first steps of the precipitation, the number of stable precipitates is varying quite linearly with time (Fig. 2). The slope of this linear relation gives a measure 


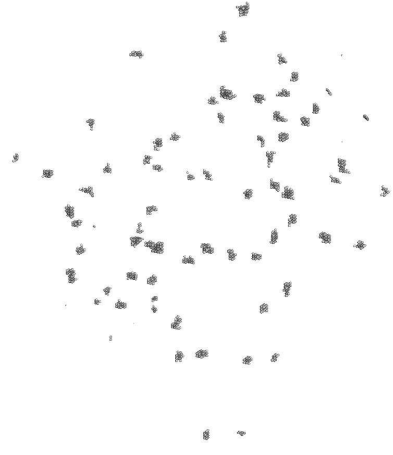

(a) $t=24 \mathrm{~ms}$

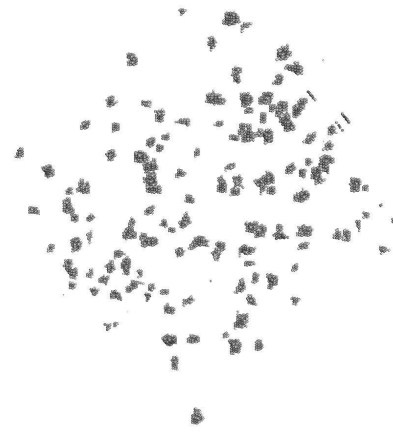

(b) $t=60 \mathrm{~ms}$

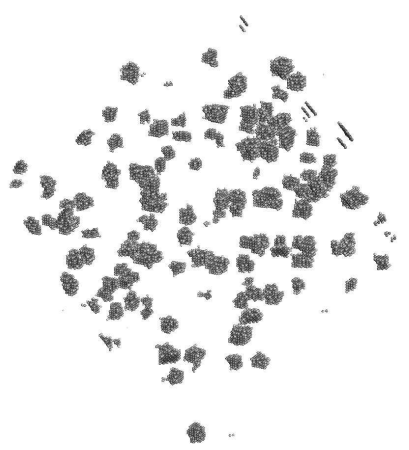

(c) $t=151 \mathrm{~ms}$

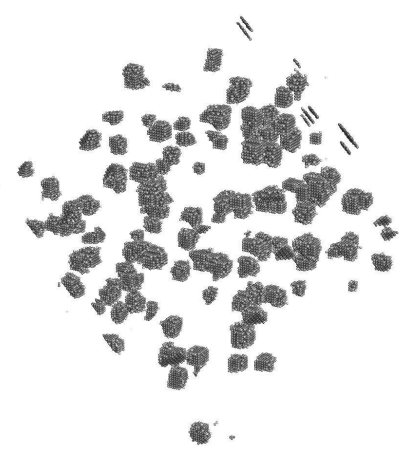

(d) $t=366 \mathrm{~ms}$

FIG. 1: Monte Carlo simulation of the kinetics of precipitation of $\mathrm{Al}_{3} \mathrm{Sc}$ for a supersaturated aluminum solid solution of nominal concentration $x_{\mathrm{Sc}}^{0}=0.005$ at $T=773 \mathrm{~K}$. The simulation box contains $8 \times 10^{6}$ lattice sites. Only Sc atoms belonging to L1 $1_{2}$ precipitates are shown. The critical size used is $n_{\mathrm{Sc}}^{*}=13$.

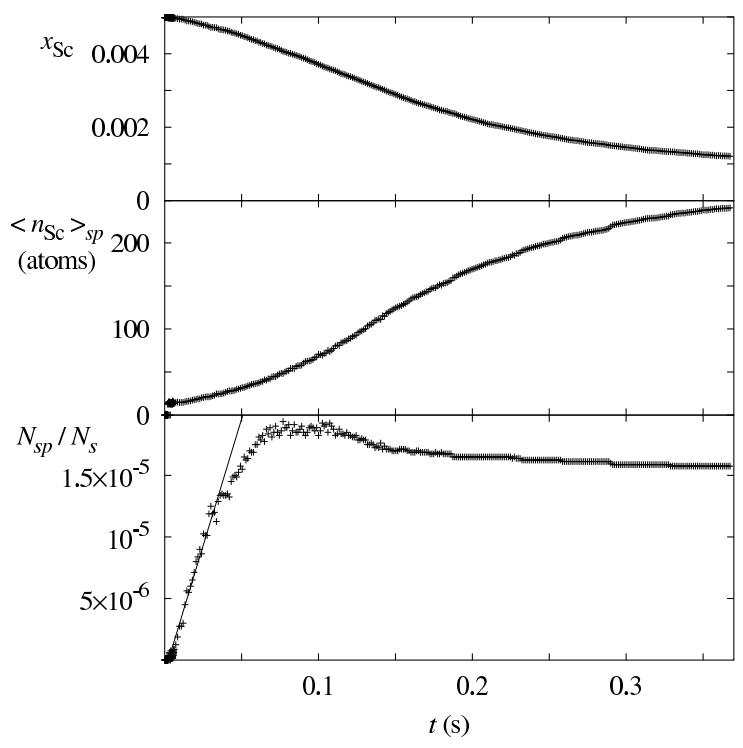

FIG. 2: Kinetics of precipitation of a supersaturated aluminum solid solution of nominal concentration $x_{\mathrm{Sc}}^{0}=0.005$ at $T=773 \mathrm{~K}$ : evolution with time of the number $N_{s p}$ of stable precipitates in the simulation box (normalized by the number of lattice sites $N_{s}$ ), of stable precipitate average size $\left.<n_{\mathrm{Sc}}\right\rangle_{s p}$, and of Sc concentration $x_{\mathrm{Sc}}$ in the solid solution. The critical size used to discriminate stable precipitates from sub-critical clusters is $n_{\mathrm{Sc}}^{*}=13$. Some of the corresponding simulation configurations are shown in Fig. 1]

of the steady-state nucleation rate $J^{\text {st }}$, i.e. the number of stable precipitates appearing by time unit during the nucleation stage.

\section{CLASSICAL NUCLEATION THEORY}

In order to compare kinetic Monte Carlo simulations with classical nucleation theory, we need to define the formation free energy $\Delta G_{n}\left(x_{\mathrm{X}}^{0}\right)$ of a $\mathrm{L}_{2}$ cluster containing $n$ atoms $\left(n=4 n_{\mathrm{Zr}}\right.$ or $\left.4 n_{\mathrm{Sc}}\right)$ embedded in a solid solution of nominal concentration $x_{\mathrm{X}}^{0}$. Usually, one uses the capillary approximation and considers a volume contribution, the nucleation free energy $\Delta G^{n u c}\left(x_{\mathrm{X}}^{0}\right)$, and a surface contribution corresponding to the energy cost to create an interface between the solid solution and the $\mathrm{L} 1_{2}$ precipitate,

$$
\Delta G_{n}\left(x_{\mathrm{X}}^{0}\right)=n \Delta G^{n u c}\left(x_{\mathrm{X}}^{0}\right)+\left(\frac{9 \pi}{4}\right)^{1 / 3} n^{2 / 3} a^{2} \bar{\sigma},
$$

where $a$ is the lattice parameter and $\bar{\sigma}$ the interface free energy. For a supersaturated solution, $\Delta G_{n}\left(x_{\mathrm{X}}^{0}\right)$ shows a maximum in $n^{*}=4 n_{\mathrm{Zr}}^{*}$ or $4 n_{\mathrm{Sc}}^{*}$ corresponding to the critical size used to follow the kinetics of precipitation during the Monte Carlo simulations ( $c f$. section $\llbracket \mathrm{C}$ ). We now have to calculate the nucleation free energy $\Delta G^{n u c}\left(x_{\mathrm{X}}^{0}\right)$ and the interface free energy $\bar{\sigma}$ corresponding to the set of atomic parameters presented in the previous section.

\section{A. Nucleation free energy}

The nucleation free energy to precipitate $\mathrm{Al}_{3} \mathrm{X}(\mathrm{X} \equiv$ $\mathrm{Zr}$ or Sc) is ${ }^{3.48}$

$$
\begin{aligned}
\Delta G^{n u c}\left(x_{\mathrm{X}}^{0}\right)= & \frac{3}{4}\left(\mu_{\mathrm{Al}}\left(x_{\mathrm{X}}^{e q}\right)-\mu_{\mathrm{Al}}\left(x_{\mathrm{X}}^{0}\right)\right) \\
& +\frac{1}{4}\left(\mu_{\mathrm{X}}\left(x_{\mathrm{X}}^{e q}\right)-\mu_{\mathrm{X}}\left(x_{\mathrm{X}}^{0}\right)\right),
\end{aligned}
$$

where $\mu_{\mathrm{Al}}\left(x_{\mathrm{X}}\right)$ and $\mu_{\mathrm{X}}\left(x_{\mathrm{X}}\right)$ are the chemical potentials of respectively $\mathrm{Al}$ and $\mathrm{X}$ components in the solid solution 


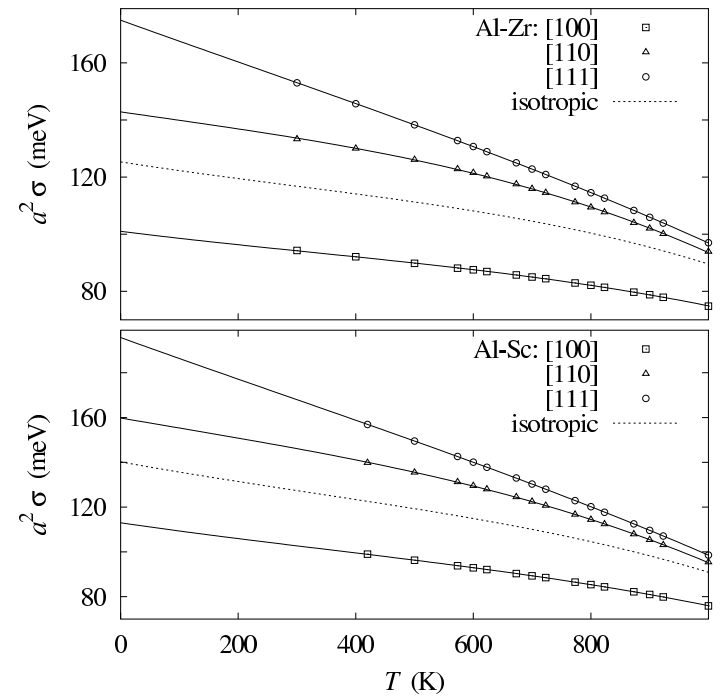

FIG. 3: Dependence with temperature of the free energies of the solid solution / $\mathrm{Al}_{3} \mathrm{Zr}$ (top) and solid solution / $\mathrm{Al}_{3} \mathrm{Sc}$ (bottom) interfaces for the [100], [110], and [111] directions, and associated isotropic free energy $\bar{\sigma}$ obtained from Wulff construction.

of concentration $x_{\mathrm{X}}, x_{\mathrm{X}}^{e q}$ is the equilibrium concentration of the solid solution, and $x_{\mathrm{X}}^{0}$ the nominal concentration. The factors $3 / 4$ and $1 / 4$ arise from the stoichiometry of the precipitating phase $\mathrm{Al}_{3} \mathrm{X}$. We use the CVM in the tetrahedron-octahedron approximation ${ }^{15.16}$ to calculate chemical potentials entering Eq. (9). This is the minimum CVM approximation that can be used with first and second nearest neighbor interactions. Within this approximation, all correlations inside the tetrahedron of first nearest neighbors and the octahedron linking the centers of the six cubic faces are included in the calculation of the chemical potentials. Usually one does not consider these correlations in the calculation of the nucleation free energy and merely uses the Bragg-Williams approximation to obtain $\Delta G^{n u c}$ but we will see in section IVB that this leads to discrepancies between results of atomic simulations and predictions of classical nucleation theory.

\section{B. Interface free energy}

\section{Plane interfaces}

We calculate interface free energies between the aluminum solid solution assumed to be at equilibrium close to the interface and the $\mathrm{L} 1_{2}$ precipitates for three different directions of the interface ([100], [110], and [111]). If phases are assumed to be pure, the different interface energies are simply related by the equation

$$
\sigma_{100}=\frac{1}{\sqrt{2}} \sigma_{110}=\frac{1}{\sqrt{3}} \sigma_{111}=\frac{\omega^{(2)}}{a^{2}} .
$$

At finite temperature, one has to consider that the solid solution is not pure $\mathrm{Al}$ and that the $\mathrm{L}_{2}$ structure differs from $\mathrm{Al}_{3} \mathrm{X}$ stoichiometry. Moreover, to minimize the energy cost due to the interface, concentrations and order parameters of planes near the interface can differ from those in the bulk. So as to take into account such a relaxation, we calculate these interface free energies within the Bragg-Williams approximation. A better statistical approximation based on CVM is too cumbersome and we only check for the [100] direction that we obtain the same value of the free energy in the whole range of temperatures with a CVM calculation in the tetrahedron approximation.

At finite temperature, we still observe that $\sigma_{100}<$ $\sigma_{110}<\sigma_{111}$ (Fig [3). Nevertheless, as the relaxation is small for the interface in the [100] direction and important for the [111] direction, the difference between interface energies is decreasing with temperature. This indicates that precipitates are becoming more isotropic at higher temperatures. Using Wulff construction ${ }^{48.49}$ to determine the precipitate equilibrium shape, we find that precipitates will mainly show facets in the [100] directions and that facets in the [110] and [111] directions are small but becoming more important with increasing temperature. Comparing these predicted equilibrium shape with the ones observed during the atomic simulations, we find a good agreement (Fig. (4): at low temperatures $(T \sim 723 \mathrm{~K})$, precipitates are cubic with sharp [100] interfaces, whereas at higher temperatures interfaces are not so sharp. For $\mathrm{Al}_{3} \mathrm{Sc}$, Marquis and Seidman ${ }^{23}$ experimentally observed precipitates showing facets in the [100], [110], and [111] directions at $T=573 \mathrm{~K}$, with [100] facets tending to disappear at high temperatures. This is well reproduced by our atomic model, the main difference being that the experimentally observed [100] facets are less important compared to the other ones than in our study.

Asta et $a l^{50}$ used a cluster expansion of ab-initio calculations to obtain the same interface energies in Al-Sc system. The energies they got are higher than ours: $a^{2} \sigma_{100}$ is varying from 167 to $157 \mathrm{meV}$ between $0 \mathrm{~K}$ and the melting temperature $\left(T^{m e l}=934 \mathrm{~K}\right)$ and $a^{2} \sigma_{111}$ from 233 to $178 \mathrm{meV}$. The difference could be due to the limited range of our interactions compared to Asta's ones. This could explain too why the interface free energies we obtain are decreasing more rapidly with temperature especially in the [111] direction ${ }^{51}$.

Hyland et $a l^{52}$ also calculated interface free energies with an empirical potential for Al-Sc, but the energies they obtained are really low compared to ours and Asta's ones as well as compared to the isotropic interface free energy they measured ${ }^{22}$. Some of the discrepancy can be due to relaxations of atomic positions which are considered in their study and are missing in our. It may also 


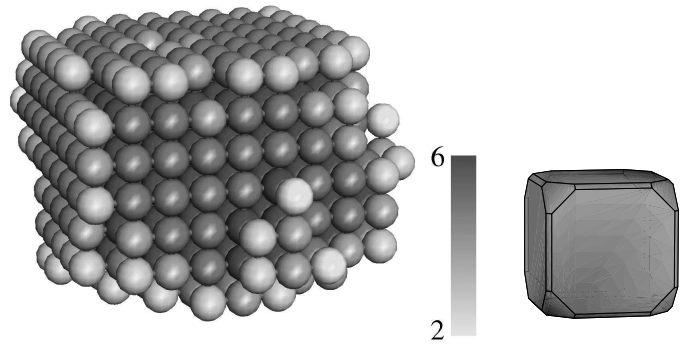

(a) $T=723 \mathrm{~K}$
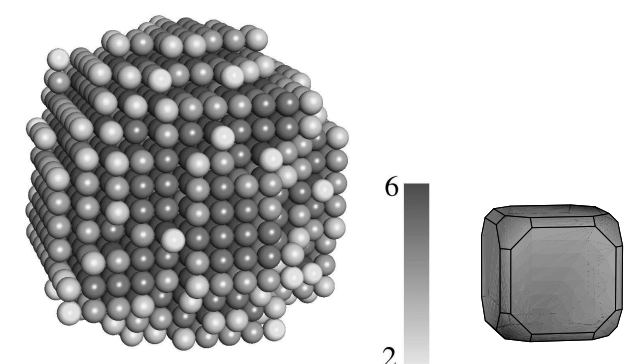

(b) $T=873 \mathrm{~K}$

FIG. 4: $\mathrm{Al}_{3} \mathrm{Sc}$ precipitate observed during Monte Carlo simulations at different temperatures and corresponding Wulff construction obtained from the interface free energies calculated at the same temperatures. For Monte Carlo simulations, only Sc atoms are shown. Atom color corresponds to the number of Sc atoms as second nearest neighbors. For a [100] interface, it should be 5, for a [110] 4, and for a [111] 3 .

indicate that the potential they used is not really well suited to describe solid solution $/ \mathrm{Al}_{3} \mathrm{Sc}$ interfaces.

\section{Average interface free energy}

We use the Wulff construction ${ }^{48,49}$ to define an isotropic free energy $\bar{\sigma}$ from the free energies $\sigma_{100}, \sigma_{110}$, and $\sigma_{100} . \bar{\sigma}$ is defined so as to give the same interface free energy for a spherical precipitate having the same volume as the real faceted one. Details of calculations can be found in appendix . The free energy $\bar{\sigma}$ is higher than the minimum energy $\sigma_{100}$ (Fig. (3). The ratio $\bar{\sigma} / \sigma_{100}$ is slightly lower than $(6 / \pi)^{1 / 3}$, this value corresponding to cubic precipitates showing only [100] facets.

Robson and Prangnell ${ }^{6}$ deduced from experimental observations of $\mathrm{Al}_{3} \mathrm{Zr}$ coarsening a $\mathrm{Al} / \mathrm{Al}_{3} \mathrm{Zr}$ interface free energy $\bar{\sigma}=100 \mathrm{~mJ} . \mathrm{m}^{-2}$ at $773 \mathrm{~K}$. The agreement between this value and the one deduced from our atomic model is perfect. In the same way, Hyland ${ }^{22}$ obtained from measured nucleation rates and incuba- tion times an experimental $\mathrm{Al} / \mathrm{Al}_{3} \mathrm{Sc}$ interface energy $\bar{\sigma}=94 \pm 23 \mathrm{~mJ} . \mathrm{m}^{-2}$ between 563 and $623 \mathrm{~K}$. Nevertheless, this experimental value should be considered only as an order of magnitude as experimental nucleation rates and incubation times are hard to obtain. One has to be sure that precipitates of the critical size can be observed and the difference between the interface energies deduced from the incubation times or from the nucleation rates could be due to a detection limit for small precipitates greater than the critical size. Moreover, the Sc diffusion coefficient used by Hyland in his study differs from the one which has been more recently obtained from radioactive tracer diffusion measurements 46 and this would influence too the value of the interface energy deduced from his experimental observations. With these considerations in mind, this experimental value although slightly lower than the one we calculate $\left(\sim 113 \mathrm{~mJ} . \mathrm{m}^{-2}\right)$ is in good agreement with it. This indicates that the use of Wulff construction with mean-field theory is a good way to estimate this isotropic interface free energy and that our set of atomic parameters (Tab.【) is realistic to model solid solution $/ \mathrm{Al}_{3} \mathrm{Zr}$ and $\mathrm{Al}_{3} \mathrm{Sc}$ interfaces.

\section{Cluster size distribution}

For a dilute solution, the probability to observe in the solid solution a cluster containing $n$ atoms having $\mathrm{L} 1_{2}$ structure is ${ }^{3.53}$

$$
C_{n} \sim \frac{C_{n}}{1-\sum_{j} C_{j}}=\exp \left(-\Delta G_{n} / k T\right),
$$

where the formation energy $\Delta G_{n}$ is given by Eq. (87). If the solution is supersaturated, the energy $\Delta G_{n}$ is decreasing for sizes greater than the critical size and Eq. (11) is assumed to be checked only for $n \leq n^{*}$. As this is the criterion we chose to discriminate the solid solution from the $\mathrm{L} 1_{2}$ precipitates ( $c f$. section IC), this means that only the cluster size distribution in the solid solution should obey Eq. (11) and not the size distribution of stable precipitates.

We compare the cluster size distribution given by the Eq. (11) with the ones measured in Monte Carlo simulations for different temperatures between 723 and $873 \mathrm{~K}$ and different concentrations of the solid solution in the Al-Zr (Fig. 5) as well as Al-Sc systems, both systems leading to the same conclusions.

For stable solid solutions $\left(x_{\mathrm{X}}^{0}<x_{\mathrm{X}}^{e q}\right)$, all energetic contributions entering $\Delta G_{n}$ are positive and the cluster critical size is not defined. Therefore, one expects Eq. (11) to be obeyed for all values of $n$. A comparison with Monte Carlo simulations shows a good agreement. The comparison can only be made for small clusters: the probability to observe large clusters in the simulations is too low to obtain statistical information on their distribution in a reasonable amount of computational time. This is interesting to note that for a solid solution having a concentration equal to the solubility limit $\left(x_{\mathrm{X}}^{0}=x_{\mathrm{X}}^{e q}\right)$, the 


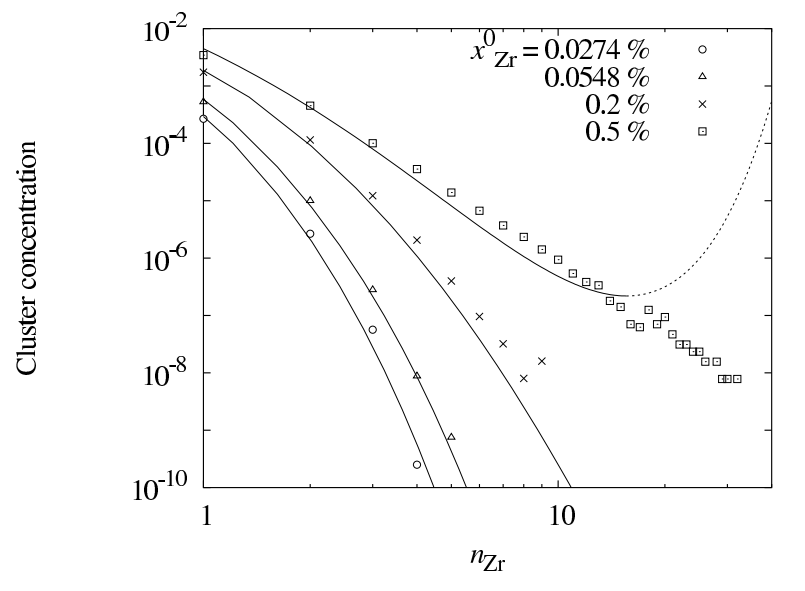

FIG. 5: Dependence with the nominal concentrations $x_{\mathrm{Zr}}^{0}$ of the cluster size distributions of an aluminum solid solution at $T=773 \mathrm{~K}$. At this temperature, the solubility limit is $x_{\mathrm{Zr}}^{e q}=5.48 \times 10^{-4}$. Lines correspond to prediction of classical nucleation theory combined with CVM calculation and symbols to Monte Carlo simulations.

prediction [11 of the cluster size distribution is still correct. As the nucleation free energy is null for this concentration, the only contribution to $\Delta G_{n}$ arises from the interface. This shows that our estimation of the interface free energy $\bar{\sigma}$ is coherent with its use in Eq. (11) and that the capillary approximation gives a good description of the solid solution thermodynamics.

For low supersaturated solid solution (for instance, on Fig. [5] $x_{\mathrm{Zr}}^{0}=0.2 \%$ ), we observe a stationary state during Kinetic Monte Carlo simulations: the computational time to obtain a stable $\mathrm{L}_{2}$ cluster is too high and the solid solution remains in its metastable state. Therefore, we can still measure the cluster size distribution during the simulations. The agreement with Eq. (11) is still correct (Fig. (5). One should notice that now, the critical size being defined, the comparison is allowed only for $n \leq n^{*}$.

For higher supersaturations, the solid solution concentration $x_{\mathrm{X}}$ is decreasing meanwhile stable precipitates appear ( $c f$. kinetics of precipitation of $\mathrm{Al}_{3} \mathrm{Sc}$ in Fig. (2). This involves that the nucleation free energy is decreasing in absolute value and that the critical size $n^{*}$ is increasing. At each step we have to re-calculate the solid solution concentration and the critical size self-consistently by means of the definition (17) of $x_{\mathrm{X}}$ and by imposing that $\Delta G_{n}\left(x_{\mathrm{X}}\right)$ is maximum in $n^{*}$. Then we use this new value of the solid solution concentration in Eq. (11) to calculate the corresponding cluster size distribution and compare it with the kinetic Monte Carlo simulation ( $c f$. cluster size distributions in a $\mathrm{Zr}$ supersaturated aluminum solution in Fig. 6). We see that the time evolution of the cluster size distribution is well reproduced by Eq. (11) when the instantaneous concentration is used to calculate the nucleation free energy and therefore the prediction of cluster size distribution is not only verified during the nucleation

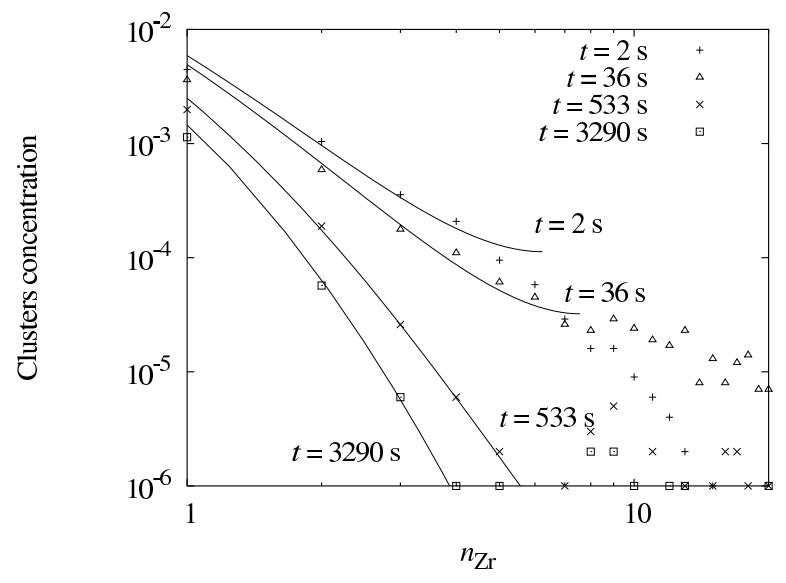

FIG. 6: Evolution with time of the cluster size distributions of an aluminum solid solution of nominal concentration $x_{\mathrm{Zr}}^{0}=1 \times 10^{-2}$ at $T=723 \mathrm{~K}$. At this temperature, the solubility limit is $x_{\mathrm{Zr}}^{e q}=2.90 \times 10^{-4}$. Symbols correspond to Monte Carlo simulations and lines to prediction of classical nucleation theory combined with CVM calculation with the following instantaneous solid solution concentrations and critical sizes: $x_{\mathrm{Zr}}=1 \times 10^{-2}, 7 \times 10^{-3}, 2.7 \times 10^{-3}$, and $1.5 \times 10^{-3}$ and $n_{\mathrm{Zr}}^{*}=7,8,18$, and 41 at respectively $t=2,36,533$, and $3290 \mathrm{~s}$.

stage but is well adapted even during the growing stage. Thus the thermodynamic description used in the classical nucleation theory is in good agreement with results of atomic simulations.

\section{Kinetic description}

\section{Diffusion}

Classical nucleation theory assumes that only monomers migrate and that larger clusters like dimers do not diffuse. We check this is the case with our atomic model by measuring during Monte Carlo simulations the diffusion coefficient associated to the gravity center of $N$ atoms $\mathrm{X}$ in pure $\mathrm{Al}$ for $2 \leq N \leq 4$. We obtain for $\mathrm{Zr}$ as well as Sc atoms that this diffusion coefficient is equal to the monomer diffusion coefficient divided by the number $N$ of $\mathrm{X}$ atoms considered. This implies the following relation

$$
\left\langle\left(\sum_{n=1}^{N} \boldsymbol{\Delta} \mathbf{r}_{\mathrm{X}_{n}}\right)^{2}\right\rangle=\sum_{n=1}^{N}\left\langle\Delta \mathbf{r}_{\mathbf{X}_{n}}{ }^{2}\right\rangle,
$$

where the brackets indicate a thermodynamic ensemble average and $\Delta \mathbf{r}_{\mathrm{X}_{n}}$ is the displacement of the atom $\mathrm{X}_{n}$ during a given time. This relation is satisfied only if there is no correlation between the displacement of the $N$ atoms X, which in other words means that cluster formed of the $N$ atoms does not diffuse. In both systems, tracer diffusion coefficient of $\mathrm{Al}$ is several order of 

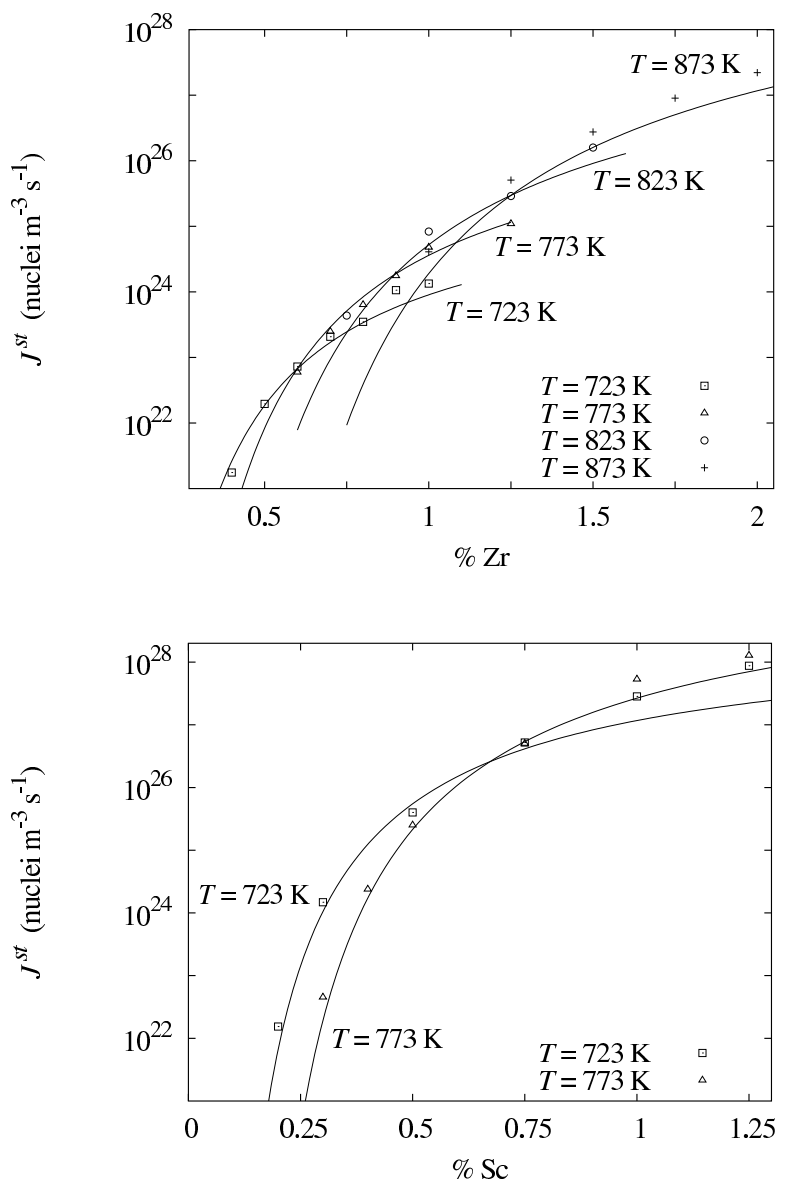

FIG. 7: Variation with nominal concentration and temperature of the steady-state nucleation rate $J^{\text {st }}$ for $\mathrm{Al}_{3} \mathrm{Zr}$ (top) and $\mathrm{Al}_{3} \mathrm{Sc}$ (bottom) precipitation. Symbols correspond to Monte Carlo simulations and lines to classical nucleation theory combined with CVM calculation.

magnitude larger than tracer diffusion coefficients of X. The relationship of Manning 54 shows that in that case the correlation factor $f_{\mathrm{Xx}}$ is almost equal to the tracer correlation factor $f_{\mathrm{X}}$ which is equivalent ${ }^{55,56}$ to Eq. (12). Thus the assumption used by classical nucleation theory of a diffusion controlled by monomers is checked for both $\mathrm{Al}-\mathrm{Zr}$ and $\mathrm{Al}-\mathrm{Sc}$ systems although interactions with vacancies are different for these two binary systems. This is not the case for all systems: when vacancies are trapped inside precipitates or at the interface with the matrix, small clusters can migrate ${ }^{12.43 .57 .58}$ which affects kinetics of precipitation.

\section{Steady-state nucleation rate}

The steady-state nucleation rate is then predicted to be given by the equation ${ }^{3}$,

$$
J^{s t}=N_{s} Z \beta^{*} \exp \left(-\Delta G^{*} / k T\right),
$$

where $N_{s}$ is the number of nucleation sites, i.e. the number of lattice sites, $\Delta G^{*}$ is the nucleation barrier and corresponds to the free energy of a precipitate of critical size $n^{*}$ as given by Eq. (8), $Z$ is the Zeldovitch factor and describes size fluctuations of precipitates around $n^{*}$,

$$
Z=\frac{\left(\Delta G^{n u c}\right)^{2}}{2 \pi\left(a^{2} \bar{\sigma}\right)^{3 / 2} \sqrt{k T}}
$$

and $\beta^{*}$ is the condensation rate for clusters of critical size $n^{*}$. Assuming the limiting step of the adsorption is the long range diffusion of $\mathrm{Zr}$ or $\mathrm{Sc}$ in the solid solution and that $\mathrm{Al}$ atoms diffuse infinitely faster than $\mathrm{Zr}$ or Sc atom, the condensation rate is ${ }^{3}$

$$
\beta^{*}=-32 \pi \frac{a^{2} \bar{\sigma}}{\Delta G^{n u c}} \frac{D_{\mathrm{X}}}{a^{2}} x_{\mathrm{X}}^{0} .
$$

Although only monomers diffuse, the concentration appearing in Eq. (15) is the nominal one as it reflects the gradient of concentration driving diffusion. Each time one $\mathrm{Zr}$ or Sc atom condensates on a cluster, three $\mathrm{Al}$ atoms condensate too on the same cluster. Thus clusters are growing from sizes $4 n$ to $4(n+1)$.

Comparing with the steady-state nucleation rate measured in Monte Carlo simulations for different temperatures and different supersaturations of the solid solution in the $\mathrm{Al}-\mathrm{Zr}$ and $\mathrm{Al}-\mathrm{Sc}$ system, we see that the classical nucleation theory manages to predict $J^{s t}$ (Fig. 17). The agreement is really good for low nominal concentrations of the solid solution $\left(x_{\mathrm{X}} \leq 1 \times 10^{-2}\right)$ and is still good for higher concentrations: there is a small discrepancy but the relative values for different temperatures at a given concentration are correctly predicted. For instance, for the nominal concentration $x_{\mathrm{Zr}}^{0}=0.01$, we obtain that the steady-state nucleation rate is higher at $T=823 \mathrm{~K}$ than at $T=773$ or $873 \mathrm{~K}$. This shows that the kinetic model used by the classical nucleation theory is checked both for $\mathrm{Al}_{3} \mathrm{Zr}$ kinetics of precipitation where there is repulsion between the vacancy and the precipitating element and for $\mathrm{Al}_{3} \mathrm{Sc}$ kinetics where there is attraction.

\section{CAPILLARY APPROXIMATION}

Although it manages to catch thermodynamics of the solid solution, the capillary approximation that we used previously can look rough. First of all, one can wonder if it is reasonable to assume spherical precipitates especially for small ones. Moreover, when counting precipitates in Monte Carlo simulations, we assume them as being stoichiometric whereas in the mean-field calculation of the nucleation free energy we include anti-site defect contribution. Another source of mistake could be the use of the Wulff construction to calculate an isotropic interface free energy: doing so, we calculate the interface free energy of the most stable precipitate and therefore neglect some configurational entropy.

In the present section, we calculate the cluster free energy without using the capillary approximation. The 
results obtained with this direct calculation are then confronted to the ones obtained with the capillary approximation. We also take the benefit of the exact results to discuss different levels of mean-field approximation used for the calculation of parameters entering in the capillary approximation.

\section{A. Direct calculation of cluster energies.}

Instead of using the capillary approximation to calculate the formation energy of $\mathrm{L}_{2}$ clusters, we can calculate this quantity exactly. This can be done, following Ref. 59, by sampling thermodynamic averages with Monte Carlo simulations so as to compute the free energy difference between a cluster of size $n$ and one of size $n+1$ at a given temperature. This method presents the drawback that a calculation is needed at every temperature of interest. We prefer calculating all coefficients entering the partition function as done in Ref. 13 and then derive the free energy at every temperature.

A L1 $1_{2}$ cluster containing $n_{\mathrm{X}} \mathrm{X}$ atoms can have different shapes which we group by classes $\alpha$ of same energy: $D_{n_{\mathrm{X}}, \alpha}$ is the number per lattice site of clusters containing $n_{\mathrm{X}} \mathrm{X}$ atoms and having the energy $H_{n_{\mathrm{X}} \alpha}=$ $n_{\mathrm{X}}\left(12 \omega^{(1)}+6 \epsilon_{\mathrm{XX}}^{(1)}-6 \epsilon_{\mathrm{AlAl}}^{(1)}+3 \epsilon_{\mathrm{XX}}^{(2)}-3 \epsilon_{\mathrm{AlAl}}^{(2)}\right)+\delta H_{n_{\mathrm{X}}, \alpha}$. Energies are defined referred to the pure $\mathrm{Al}$ reference state as the cluster energy is the energy change due to the presence of a cluster in pure Al. The free energy of a $\mathrm{L} 1_{2}$ cluster containing $n_{\mathrm{X}} \mathrm{X}$ atoms is then defined by

$$
\begin{aligned}
G_{n_{\mathrm{X}}}= & -k T \ln \left(\sum_{\alpha} D_{n_{\mathrm{X}}, \alpha} \exp \left(-H_{n_{\mathrm{X}}, \alpha} / k T\right)\right) \\
= & n_{\mathrm{X}}\left(12 \omega^{(1)}+6 \epsilon_{\mathrm{XX}}^{(1)}-6 \epsilon_{\mathrm{AlAl}}^{(1)}+3 \epsilon_{\mathrm{XX}}^{(2)}-3 \epsilon_{\mathrm{AlAl}}^{(2)}\right) \\
& -k T \ln \left(\sum_{\alpha} D_{n_{\mathrm{X}, \alpha}} \exp \left(-\delta H_{n_{\mathrm{X}}, \alpha} / k T\right)\right)
\end{aligned}
$$

Degeneracies $D_{n_{\mathrm{X}}, \alpha}$ can be computed for a given size by generating clusters with a random configuration and then by counting for each energy level $\alpha$ the number of different clusters. The obtained values are presented in table III for $\mathrm{L} 1_{2}$ clusters containing less than $9 \mathrm{X}$ atoms. For bigger clusters, the degeneracy of the different classes is becoming too high to be countable. This is important to notice that we use the same criterion to define $\mathrm{L}_{2}$ clusters as in the kinetic Monte Carlo simulations ( $c f$. section $\Pi \mathrm{C}$ ), and that $\mathrm{Zr}$ or Sc atoms belonging to a $\mathrm{L}_{2}$ cluster only have $\mathrm{Al}$ atoms as first nearest neighbors. We thus do not allow anti-site defects on the majority sub-lattice. This is not an important restriction as these defects have a high formation energy and therefore their contribution to the partition function can be neglected. As for the minority sub-lattice, anti-site defects can not be taken into account as they lead to a change of the precipitate size.
TABLE III: Degeneracies $D_{n_{\mathrm{X}}, \alpha}$ corresponding to classes of $\mathrm{L}_{2}$ clusters containing $n_{\mathrm{X}} \mathrm{X}$ atoms and having energy $H_{n_{\mathrm{X}}, \alpha}=n_{\mathrm{X}}\left(12 \omega^{(1)}+6 \epsilon_{\mathrm{XX}}^{(1)}-6 \epsilon_{\mathrm{AlAl}}^{(1)}+3 \epsilon_{\mathrm{XX}}^{(2)}-3 \epsilon_{\mathrm{AlAl}}^{(2)}\right)+$ $\delta H_{n_{\mathrm{X}}, \alpha}$ for $1 \leq n_{\mathrm{X}} \leq 9$.

\begin{tabular}{rrrrrrrr}
\hline \hline$n_{\mathrm{X}}$ & $\alpha$ & $\delta H_{n_{\mathrm{X}}, \alpha}$ & $D_{n_{\mathrm{X}}, \alpha}$ & $n_{\mathrm{X}}$ & $\alpha$ & $\delta H_{n_{\mathrm{X}}, \alpha}$ & $D_{n_{\mathrm{X}}, \alpha}$ \\
\hline \multirow{2}{*}{1} & \multirow{2}{*}{$6 \omega^{(2)}$} & 1 & 7 & 2 & $26 \omega^{(2)}$ & 878 \\
& & & & 7 & 3 & $28 \omega^{(2)}$ & 4368 \\
2 & 1 & $10 \omega^{(2)}$ & 3 & 7 & 4 & $30 \omega^{(2)}$ & 18746 \\
& & & & & & & \\
3 & 1 & $14 \omega^{(2)}$ & 15 & 8 & 1 & $24 \omega^{(2)}$ & 1 \\
& & & & 8 & 2 & $28 \omega^{(2)}$ & 306 \\
4 & 1 & $16 \omega^{(2)}$ & 3 & 8 & 3 & $30 \omega^{(2)}$ & 4829 \\
4 & 2 & $18 \omega^{(2)}$ & 83 & 8 & 4 & $32 \omega^{(2)}$ & 35926 \\
& & & & 8 & 5 & $34 \omega^{(2)}$ & 121550 \\
5 & 1 & $20 \omega^{(2)}$ & 48 & & & & \\
5 & 2 & $22 \omega^{(2)}$ & 486 & 9 & 1 & $28 \omega^{(2)}$ & 24 \\
& & & & 9 & 2 & $30 \omega^{(2)}$ & 159 \\
6 & 1 & $22 \omega^{(2)}$ & 18 & 9 & 3 & $32 \omega^{(2)}$ & 5544 \\
6 & 2 & $24 \omega^{(2)}$ & 496 & 9 & 4 & $34 \omega^{(2)}$ & 51030 \\
6 & 3 & $26 \omega^{(2)}$ & 2967 & 9 & 5 & $36 \omega^{(2)}$ & 289000 \\
& & & & 9 & 6 & $38 \omega^{(2)}$ & 803000 \\
\hline \hline
\end{tabular}

The formation energies entering Eq. (11) to calculate the cluster concentrations are the formation energies relative to the solid solution,

$$
\Delta G_{n_{\mathrm{X}}}\left(x_{\mathrm{X}}^{0}\right)=G_{n_{\mathrm{X}}}-2 n_{\mathrm{X}} \mu\left(x_{\mathrm{X}}^{0}\right),
$$

where $\mu\left(x_{\mathrm{X}}^{0}\right)=\left(\mu_{\mathrm{X}}\left(x_{\mathrm{X}}^{0}\right)-\mu_{\mathrm{Al}}\left(x_{\mathrm{X}}^{0}\right)\right) / 2$ is the effective potential, i.e. a Lagrange multiplier imposing that the nominal concentration of the solid solution is equal to the concentration of solute contained in the clusters as given by Eq. (7). As in the capillary approximation, this formation energy can be divided into a volume and an interface contribution:

$$
\begin{aligned}
\Delta G_{n_{\mathrm{X}}}\left(x_{\mathrm{X}}^{0}\right)= & 4 n_{\mathrm{X}} \Delta G^{n u c}\left(x_{\mathrm{X}}^{0}\right) \\
& +\left(36 \pi n_{\mathrm{X}}^{2}\right)^{1 / 3} a^{2} \sigma_{n_{\mathrm{X}}}
\end{aligned}
$$

where we have defined the nucleation free energy

$$
\begin{aligned}
\Delta G^{n u c}\left(x_{\mathrm{X}}^{0}\right)= & \left(12 \omega^{(1)}+6 \epsilon_{\mathrm{XX}}^{(1)}-6 \epsilon_{\mathrm{AlAl}}^{(1)}\right. \\
& \left.+3 \epsilon_{\mathrm{XX}}^{(2)}-3 \epsilon_{\mathrm{AlAl}}^{(2)}-2 \mu\left(x_{\mathrm{X}}^{0}\right)\right) / 4
\end{aligned}
$$

and the interface free energy

$$
\begin{aligned}
a^{2} \sigma_{n_{\mathrm{X}}}= & -k T\left(36 \pi n_{\mathrm{X}}^{2}\right)^{-1 / 3} \\
& \ln \left(\sum_{\alpha} D_{n_{\mathrm{X}}, \alpha} \exp \left(-\delta H_{n_{\mathrm{X}}, \alpha} / k T\right)\right) .
\end{aligned}
$$

All information concerning the solid solution, i.e. its nominal concentration, is contained in the nucleation free energy whereas the interface free energy is an intrinsic 


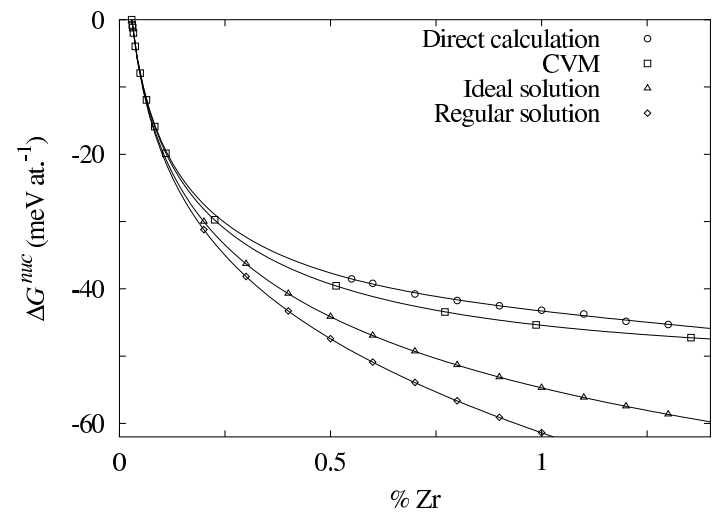

FIG. 8: Variation with the nominal concentration $x_{\mathrm{Zr}}^{0}$ of the nucleation free energy $\Delta G^{n u c}$ at $T=723 \mathrm{~K}$ obtained with different approximation: direct calculation of the cluster formation free energy (Eq. (19) ) or capillary approximation with the nucleation free energy given by the CVM calculation, the ideal solid solution model, and the regular solid solution model.

property of clusters, which was already the case with the capillary approximation. The main difference is that now the interface free energy depends on cluster size. Perini et al ${ }^{59}$ show that this size dependence can be taken into account in the capillary approximation by adding terms to the series (8) of the formation energy reflecting line and point contributions.

We compare the nucleation free energy obtained from this direct calculation of the cluster formation energies with the one that we previously calculated with CVM in section $11 \mathrm{~A}$ (Fig. 8). The direct calculation leads to a slightly lower nucleation free energy in absolute value than the CVM one. This mainly arises from the neglect of excluded volume between the different clusters in the direct calculation. Nevertheless, the agreement is correct for all temperatures and for both $\mathrm{Al}-\mathrm{Zr}$ and $\mathrm{Al}-\mathrm{Sc}$ systems. This shows that these two approaches used to describe thermodynamics of the solid solution, i.e. the mean-field and the cluster descriptions, are consistent.

The interface free energy defined by Eq. (20) is decreasing with cluster size, the variation becoming more important at higher temperatures (Fig. 9). The asymptotic limit is smaller than the interface free energy $\bar{\sigma}$ that we calculated in section [IIB using Wulff construction and Bragg-Williams approximation. This is quite natural as Wulff construction predicts the cluster shape costing least energy. We are thus missing some configurational entropy by using it to compute an interface free energy $\bar{\sigma}$ and we overestimate $\bar{\sigma}$. This error can be neglected at low temperature $(T \leq 773 \mathrm{~K})$ where precipitates show sharp interfaces but it increases with temperature when precipitate shapes are becoming smoother.

We use this direct calculation of the cluster formation free energy (Eq. (16) and (17)) to predict cluster size distributions in the solid solution and compare the re-

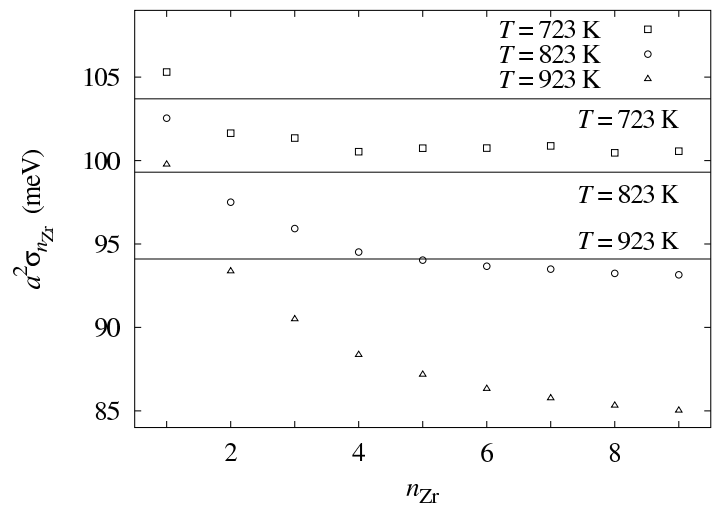

FIG. 9: Variation with the cluster size $n_{\mathrm{Zr}}$ of the interface free energy between the solid solution and $\mathrm{Al}_{3} \mathrm{Zr}$. Symbols correspond to $\sigma_{n_{\mathrm{Zr}}}$ as given by the direct calculations of the cluster formation free energy (Eq. (20) ) and lines to $\bar{\sigma}$, i.e. the Bragg-Williams calculation combined with the Wulff construction.

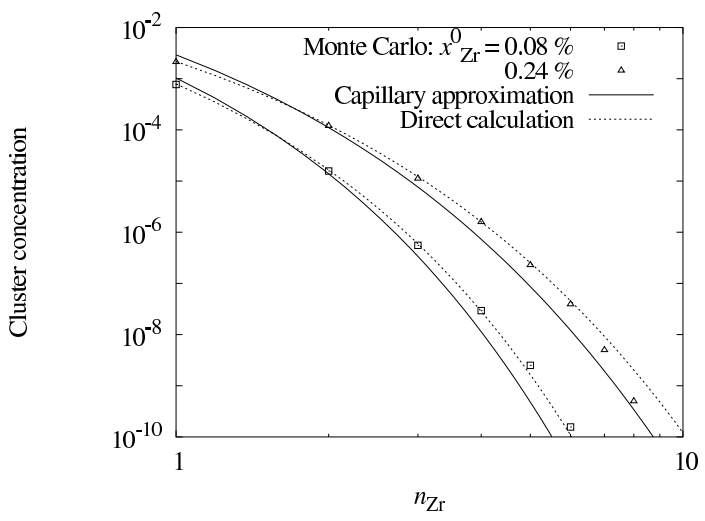

FIG. 10: Cluster size distribution of two aluminum solid solutions of nominal concentrations $x_{\mathrm{Zr}}^{0}=8 \times 10^{-4}$ and $2.4 \times 10^{-3}$ at $T=873 \mathrm{~K}$. At this temperature, the solubility limit is $x_{\mathrm{Zr}}^{e q}=1.6 \times 10^{-3}$. Symbols correspond to Monte Carlo simulations and lines to prediction of classical nucleation theory as given by Eq. (11). To evaluate the cluster free energy of formation, we use the capillary approximation (Eq. (8)) with the nucleation free energy given by CVM for the continuous line and the direct calculation (Eq. (16) and (17) for the dashed line.

sults with the distributions obtained with the capillary approximation (Eq. (8) ) combined to the CVM calculation. These two models lead to similar distributions (Fig. 10), indicating that the associated thermodynamic descriptions are consistent. Nevertheless, the distribution predicted by the direct calculation better reproduces the ones measured during the Monte Carlo simulations. Thus, the capillary model is good to describe thermodynamics of the solid solution but it can be improved.

Comparing the steady-state nucleation rates predicted by the two thermodynamic models with the ones mea- 


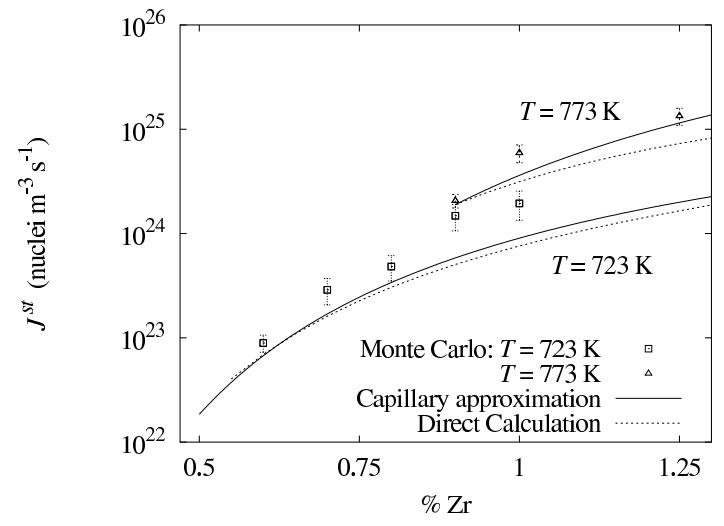

FIG. 11: Variation with nominal concentration $x_{\mathrm{Zr}}^{0}$ and temperature of the steady-state nucleate rate $J^{s t}$ for $\mathrm{Al}_{3} \mathrm{Zr}$. Solid lines correspond to prediction of the classical nucleation theory when using the capillary approximation with the CVM calculation of the nucleation free energy and dashed lines when using the direct calculation of the cluster formation free energy. Symbols are measurements in Monte Carlo simulations. The errorbars correspond to the uncertainty on the measurements of $J^{s t}$ due to the choice of the critical size corresponding to each energetic model.

sured in Monte Carlo simulations (Fig. 11), we do not obtain any improvement by using the direct calculation of cluster energy instead of the capillary approximation. For low supersaturations, both models are in reasonable agreement with Monte Carlo simulations whereas for higher supersaturations discrepancies appear. The direct calculation leads to a slightly lower nucleation rate than the capillary approximation. This mainly arises from a difference of the critical size: $n_{\mathrm{X}}^{*}$ is usually 1 atom greater with the direct calculation than with the capillary approximation. As the use of the direct calculation improves the agreement for the cluster size distribution, the discrepancy observed at high supersaturations is not due to a bad description of the solid solution thermodynamics but may arise from limitations of classical nucleation theory itself. The assumption of a constant flux between the different size classes made by this theory to solve the rate equations associated to the cluster size evolution may not apply at high supersaturations. This can be seen in our atomic simulations by the fact that, for these supersaturations, the linear domain observed for the variation with time of the number of precipitates and used to define the steady-state nucleation rate is more restricted than in the low supersaturation case shown on Fig. 1] One could try to improve the agreement with atomic simulations by using more sophisticated mesoscopic models like cluster dynamics 60.61 .62 .63 which do not need such a kinetic assumption to solve the rate equations. Another improvement that could be made to classical nucleation theory is to consider the variation with the nominal concentration of the diffusion coefficient of $\mathrm{X}$ atoms which would lead to a diffusion coefficient different from the impurity one that we use.

\section{B. Other mean-field approximation}

Usually, one does not calculate the nucleation free energy with CVM as we did in section IIIA but one uses simpler mean-field approximation to evaluate the chemical potentials entering Eq. (9) of $\Delta G^{n u c}$. We test these other approximations and see if they are reliable to be used with classical nucleation theory.

The easiest approximation that can be used is the ideal solid solution model in which one keeps only the configurational entropy contribution in the expression of chemical potentials and calculates this term within the BraggWilliams approximation. This leads to the following expression

$$
\begin{aligned}
\Delta G_{\text {ideal }}^{n u c}\left(x_{\mathrm{X}}^{0}\right)= & \frac{3}{4} \ln \left(\frac{1-x_{\mathrm{X}}^{e q}}{1-x_{\mathrm{X}}^{0}}\right) \\
& +\frac{1}{4} \ln \left(\frac{x_{\mathrm{X}}^{e q}}{x_{\mathrm{X}}^{0}}\right) .
\end{aligned}
$$

The exact expression of the nucleation free energy, i.e. with the enthalpic contribution, can be calculated within the Bragg-Williams approximation too. This is called the regular solid solution model and gives

$$
\begin{aligned}
\Delta G_{\mathrm{BW}}^{n u c}\left(x_{\mathrm{X}}^{0}\right)= & \Delta G_{\text {ideal }}^{n u c}\left(x_{\mathrm{X}}^{0}\right)+\Omega\left[\frac{3}{4}\left(x_{\mathrm{X}}^{e q 2}-x_{\mathrm{X}}^{0}{ }^{2}\right)\right. \\
& \left.+\frac{1}{4}\left(\left(1-x_{\mathrm{X}}^{e q}\right)^{2}-\left(1-x_{\mathrm{X}}^{0}\right)^{2}\right)\right] .
\end{aligned}
$$

Comparing all different mean-field approximations used to evaluate the nucleation free energy (Fig. 8), we see that for low supersaturations all approximations are close, but that for an increasing nominal concentration of the solid solution discrepancies between the different approximations are becoming more important. Both ideal and regular solid solution models overestimate the nucleation free energy compared to the CVM and the direct calculations. The discrepancy is even worse when all contributions, i.e. the enthalpic and entropic ones, are considered in the Bragg-Williams approximation. Thus, when the supersaturations is becoming too important, the Bragg-Williams approximation seems too rough to give a reliable approximation of the nucleation free energy.

This becomes clear when combining these approximations of $\Delta G^{n u c}$ with classical nucleation theory to predict cluster size distributions. The ideal and the regular solid solution models completely fail for high supersaturations to predict the cluster size distributions observed during Monte Carlo simulations (Fig. 12). The predicted critical size $n_{\mathrm{X}}^{*}$ is too small as it corresponds to a cluster size in the observed stationary distribution and the predicted probabilities for each cluster size are too high compared to the observed ones. As the prediction of the steady-state nucleation rate by classical 


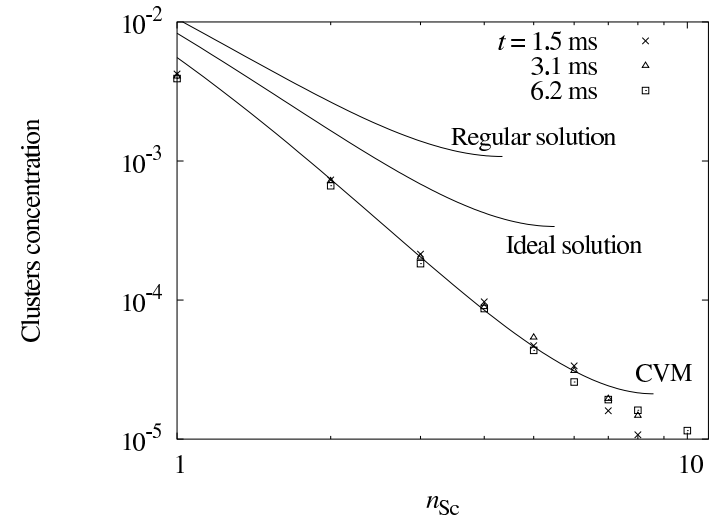

FIG. 12: Cluster size distribution of an aluminum solid solution of nominal concentration $x_{\mathrm{Sc}}^{0}=7.5 \times 10^{-3}$ at $T=773 \mathrm{~K}$. Symbols correspond to Monte Carlo simulations and lines to prediction of classical nucleation theory with the different mean field approximations of the nucleation free energy.

nucleation theory is based on the predicted size distribution, the ideal solution model and the Bragg-Williams approximation lead to an overestimation of $J^{s t}$ too. Thus the use of CVM to calculate nucleation free energy really improves agreement with atomic simulations compared to more conventional mean-field approximations. This arises from the fact that order effects are not taken into account in Bragg-Williams approximation whereas they are in CVM. These order effects correspond to a strong attraction for first nearest neighbors and a strong repulsion for second nearest neighbors between $\mathrm{Al}$ and $\mathrm{Zr}$ atoms as well as $\mathrm{Al}$ and $\mathrm{Sc}$ atoms. They are the reason at the atomic scale why a supersaturated aluminum solid solution evolves to lead to the precipitation of a $\mathrm{L}_{2}$ compound. Therefore one must fully consider these order effects when modelling kinetics of precipitation.

\section{CONCLUSIONS}

We built an atomic kinetic model for Al-Zr and Al-Sc binary systems so as to be as close as possible to the real systems. Thanks to this model, we were able to simulate at an atomic scale kinetics of precipitation of the $\mathrm{L}_{2}$ ordered compounds $\mathrm{Al}_{3} \mathrm{Zr}$ and $\mathrm{Al}_{3} \mathrm{Sc}$.

From this atomic model we deduced the corresponding interface and nucleation free energies which, with the diffusion coefficients, are the only parameters required by mesoscopic models like classical nucleation theory. When CVM is used to calculate the nucleation free energy we showed that the capillary approximation leads to a satisfying thermodynamic description of the solid solution. If one wants to improve this description, one can calculate directly formation free energies of the different size clusters. This leads to a better description of the thermodynamic behavior of the solid solution, as the agreement on cluster size distribution is better, but it does not dramatically change predictions of the classical nucleation theory. This shows that the capillary approximation is reasonable. From the kinetic point of view, classical nucleation theory assumes that evolution of the different clusters is governed by the long range diffusion of monomers. For $\mathrm{Al}-\mathrm{Zr}$ and Al-Sc systems, it appears to be a good assumption as we checked that di-, tri-, and 4-mers do not diffuse and that the steady-state nucleation rates measured in Monte Carlo simulations are in good agreement with predictions of the classical nucleation theory. Discrepancies appear at higher supersaturations which may be due to the dependence of the diffusion coefficient with the solute concentration of the metastable solid solution or to the limits of the classical nucleation theory which requires the nucleation regime to be separated from the growth regime. Nevertheless, the nucleation model was built on purpose to predict kinetics at low supersaturations for which kinetic Monte Carlo simulations are not tractable.

On the other hand, when one uses less sophisticated mean field approximation than CVM like the BraggWilliams approximation to calculate the nucleation free energy, predictions of the classical nucleation theory completely disagrees with Monte Carlo simulations, especially when supersaturations are too high. This shows that short range order effects which are naturally considered in CVM must be taken into account so as to build a kinetic mesoscopic model based on a reasonable physical description. This is expected to be the case for all systems where order effects are important and thus for systems leading to the precipitation of an ordered compound.

\section{Acknowledgments}

The authors are grateful to Dr J. Dalla Torre, Dr B. Legrand, Dr F. Soisson, and Dr G. Martin for their invaluable help and advice on many aspect of Monte Carlo simulations and classical nucleation theory. They would like to thanks too Dr Y. Le Bouar and Dr A. Finel for helpful discussions on interface free energy and low temperature expansions. This work was funded by the joint research program "Precipitation" between Pechiney, Usinor, CNRS, and CEA.

\section{APPENDIX: WULFF CONSTRUCTION}

We use the Wulff construction ${ }^{48.49}$ so as to define an isotropic interface free energies $\bar{\sigma}$ from the free energies $\sigma_{100}, \sigma_{110}$, and $\sigma_{111}$. This construction allows us to determine precipitate real shape and to associate with it $\bar{\sigma}$ which corresponds to the same interface energy for a spherical precipitate having the same volume.

$\mathrm{Al}_{3} \mathrm{X}$ precipitates will show facets in the [100], [110], 
and [111] directions if the following conditions are met:

$$
\begin{gathered}
\sqrt{2} / 2 \sigma_{100}<\sigma_{110}<\sqrt{2} \sigma_{100}, \\
\sqrt{6} / 3 \sigma_{110}<\sigma_{111}<2 \sqrt{6} / 3 \sigma_{110}-\sqrt{3} / 3 \sigma_{100} .
\end{gathered}
$$

For $\mathrm{Al}_{3} \mathrm{Zr}$ and $\mathrm{Al}_{3} \mathrm{Sc}$, with the set of parameters given by table $\llbracket$ this is true for all temperatures. Each facet surface will then be proportional to

$$
\begin{aligned}
\Gamma_{100}= & 4\left(\sigma_{100}-\sqrt{2} \sigma_{110}\right)^{2} \\
& -2\left(\sigma_{100}-2 \sqrt{2} \sigma_{110}+\sqrt{3} \sigma_{111}\right)^{2}, \\
\Gamma_{110}= & 2 \sqrt{2}\left(-2 \sigma_{100}+\sqrt{2} \sigma_{110}\right) \\
& \left(\sqrt{2} \sigma_{110}-\sqrt{3} \sigma_{111}\right) \\
\Gamma_{111}= & 3 \sqrt{3} / 2\left(-\sigma_{100}^{2}-2 \sigma_{110}^{2}+\sigma_{111}^{2}\right) \\
& +3 / 2 \sigma_{100}\left(4 \sqrt{6} \sigma_{110}-6 \sigma_{111}\right) .
\end{aligned}
$$

Considering a spherical precipitate with the same volume and the same interface energy, one gets

$$
\bar{\sigma}=\sqrt[3]{\frac{1}{4 \pi}\left(6 \sigma_{100} \Gamma_{100}+12 \sigma_{110} \Gamma_{110}+8 \sigma_{111} \Gamma_{111}\right)} .
$$

* Electronic address: emmanuel.clouet@cea.fr

${ }^{1}$ F. Soisson, in 64 .

${ }^{2}$ P. Bellon, in ${ }^{64}$.

${ }^{3}$ G. Martin, in Solid State Phase Transformation in Metals and Alloys (Les Éditions de Physique, Orsay, France, 1978), pp. 337-406.

${ }^{4}$ R. Wagner and R. Kampmann, in Materials Science and Technology, a Comprehensive Treatment, edited by R. W. Cahn, P. Haasen, and E. J. Kramer (VCH, Weinheim, 1991), vol. 5, chap. 4, pp. 213-303.

5 A. Deschamps and Y. Bréchet, Acta Mater. 47, 293 (1999).

6 J. D. Robson and P. B. Prangnell, Acta Mater. 49, 599 (2001).

7 M. J. Stowell, Materials Science and Technology 18, 139 (2002).

8 P. Maugis, D. Gendt, S. Lanteri, and P. Barges, in Defect and Diffusion Forum, edited by Y. Limoge and J. L. Bocquet (Scitec Publications, Switzerland, 2001), vol. 194199, pp. 1767-1772.

9 R. A. Ramos, P. A. Rikvold, and M. A. Novotny, Phys. Rev. B 59, 9053 (1999).

10 M. A. Novotny, P. A. Rikvold, M. Kolesik, D. M. Townsley, and R. A. Ramos, J. Non-Cryst. Solids 274, 356 (2000).

11 V. A. Shneidman, K. A. Jackson, and K. M. Beatty, Phys. Rev. B 59, 3579 (1999).

12 F. Soisson and G. Martin, Phys. Rev. B 62, 203 (2000).

13 F. Berthier, B. Legrand, J. Creuze, and R. Tétot, J. Electroanal. Chem. 561, 37, 562, 127 (2004).

14 D. de Fontaine, Solid State Phys. 47, 33 (1994).

15 R. Kikuchi, Phys. Rev. 81, 988 (1951).

16 J. M. Sanchez and D. de Fontaine, Phys. Rev. B 17, 2926 (1978).

17 T. Mohri, J. M. Sanchez, and D. de Fontaine, Acta Metall. 33, 1171 (1985).

18 A. Finel, in Statics and Dynamics of Phase Transformations, edited by P. E. A. Turchi and A. Gonis (Plenum Press, New York, 1994), pp. 495-540.

19 P. Villars and L. D. Calvert, Pearson's Handbook of Crystallographic Data for Intermetallic Phases (American Society for Metals, 1985).

20 N. Ryum, Acta Metall. 17, 269 (1969).
21 E. Nes, Acta Metall. 20, 499 (1972).

22 R. W. Hyland, Metall. Trans. A 23, 1947 (1992).

23 E. A. Marquis and D. N. Seidman, Acta Mater. 49, 1909 (2001).

${ }^{24}$ G. M. Novotny and A. J. Ardell, Materials Science and Engineering A318, 144 (2001).

25 E. Clouet, J. M. Sanchez, and C. Sigli, Phys. Rev. B 65, 094105 (2002).

${ }^{26}$ E. Clouet and M. Nastar, in Third International Alloy Conference, edited by A. Gonis, P. E. A. Turchi, A. Meike, and K. Rajan (Lisbon, 2002, to be published).

27 M. Methfessel and M. van Schilfgaarde, Phys. Rev. B 48, 4937 (1993).

28 J. P. Perdew, K. Burke, and M. Ernzerhof, Phys. Rev. Lett. 77, 3865 (1996).

29 M. Asta and V. Ozolins, Phys. Rev. B 64, 094104 (2001).

${ }^{30}$ V. Ozolins and M. Asta, Phys. Rev. Lett. 86, 448 (2001).

31 F. Ducastelle, Order and Phase Stability in Alloys (NorthHolland, Amsterdam, 1991).

32 J. Murray, J. Phase Equilib. 19, 380 (1998).

33 R. Hultgren, P. D. Desai, D. T. Hawkins, M. Gleiser, K. K. Kelley, and D. D. Wagman, Selected Values of the Thermodynamic Properties of the Elements (Am. Soc. Metals, Metals Park, Ohio, 1973).

${ }^{34}$ P. Ehrhart, P. Jung, H. Schultz, and H. Ullmaier, in Landolt-Börnstein, New Series, Group III, edited by H. Ullmaier (Springer-Verlag, Berlin, 1991), vol. 25.

35 O. Le Bacq, F. Willaime, and A. Pasturel, Phys. Rev. B 59, 8508 (1999).

36 Y. Miura, C.-H. Joh, and T. Katsube, Materials Science Forums 331-337, 1031 (2000).

37 J. P. Simon, Phys. Stat. Sol. (a) 41, K107 (1977).

38 T. Hoshino, R. Zeller, and P. H. Dederichs, Phys. Rev. B 53, 8971 (1996).

39 K. M. Carling, G. Wahnström, T. R. Mattson, A. E. Mattson, N. Sandberg, and G. Grimvall, Phys. Rev. Lett. 85, 3862 (2000).

40 K. M. Carling, G. Wahnström, T. R. Mattson, N. Sandberg, and G. Grimvall, Phys. Rev. B 67, 054101 (2003).

41 M. Nastar, P. Bellon, G. Martin, and J. Ruste, in Mat. Res. Soc. Symp. Proc. (1997), vol. 481, p. 383. 
42 A. Van der Ven, G. Ceder, M. Asta, and P. D. Tepesch, Phys. Rev. B 64, 184307 (2001).

43 Y. Le Bouar and F. Soisson, Phys. Rev. B 65, 0914103 (2002).

44 H. Bakker, H. P. Bonzel, C. M. Bruff, M. A. Dayananda, W. Gust, J. Horvth, I. Kaur, G. Kidson, A. D. LeClaire, H. Mehrer, et al., in Landolt-Börnstein, New Series, Group III, edited by H. Mehrer (Springer-Verlag, Berlin, 1990), vol. 26 .

45 T. Marumo, S. Fujikawa, and K. Hirano, Keikinzoku - J. Jpn. Inst. Light Met. 23, 17 (1973).

46 S. I. Fujikawa, Defects and Diffusion Forum 143-147, 115 (1997).

47 J. Dalla Torre (2002), private communication.

48 D. A. Porter and K. E. Easterling, Phase Transformations in Metals and Alloys (Chapman \& Hall, London, 1992).

49 J. W. Christian, The Theory of Transformations in Metals and Alloys - Part I: Equilibrium and General Kinetic Theory (Pergamon Press, Oxford, 1975).

50 M. Asta, S. M. Foiles, and A. A. Quong, Phys. Rev. B 57, 11265 (1998).

51 M. Sluiter and Y. Kawazoe, Phys. Rev. B 54, 10381 (1996).

52 R. W. Hyland, M. Asta, S. M. Foiles, and C. L. Rohrer,
Acta Mater. 46, 3667 (1998).

53 J. Frenkel, Kinetic Theory of Liquids (Dover Publications, New York, 1955).

54 J. R. Manning, Phys. Rev. B 4, 1111 (1971).

55 A. R. Allnatt, J. Phys. C: Solid State Phys. 15, 5605 (1982).

56 A. R. Allnatt and A. B. Lidiard, Atomic Transport in Solids (Cambridge University Press, 1993).

57 M. Athènes, P. Bellon, and G. Martin, Acta Mater. 48, 2675 (2000).

58 J. M. Roussel and P. Bellon, Phys. Rev. B 63, 184114 (2001).

59 A. Perini, G. Jacucci, and G. Martin, Phys. Rev. B 29, 2689 (1984).

60 K. Binder and D. Stauffer, Adv. Phys. 25, 343 (1976).

61 P. Mirold and K. Binder, Acta Metall. 25, 1435 (1977).

62 K. Binder, Rep. Prog. Phys. 50, 783 (1987).

63 P. Guyot, L. Lae, and C. Sigli, in 64.

64 A. Finel, D. Mazière, and M. Veron, eds., Thermodynamics, Microstructures, and Plasticity, vol. 108 of NATO Science Series: II: Mathematics, Physics and Chemistry (Kluwer Academic, Dordrecht, 2002). 